\title{
Effect of microemulsion on foam stability
}

\author{
Stephen Yin-Chyuan Jong ${ }^{1}$ (D) $\cdot$ Quoc Phuc Nguyen ${ }^{1}$
}

Received: 29 June 2017 / Accepted: 9 May 2018 / Published online: 25 May 2018

c) Springer-Verlag GmbH Germany, part of Springer Nature 2018

\begin{abstract}
Microemulsion-foam interactions are significant in the low tension gas process, an emerging enhanced oil recovery method. As oil-water-surfactant systems are subjected to various salinity environments and microemulsion phase behavior varies, foam strength has also been observed to vary. This may be due to the action of oil-swollen micelles within liquid lamellae. Winsor Type I microemulsions were characterized according to surface tension, oil content, oil-swollen micelle size, and viscosity. Their impact on foam stability was quantified via dynamic Bikerman-style glass column tests and static decay tests in a physical rock network microfluidic chip to observe behavior and trends across scales. Foam stability tests demonstrated up to $90 \%$ decrease in stability with similar trends at both scales as oil-swollen micelle diameter increased from 9.30 to $27.08 \mathrm{~nm}$ and concentration decreased over $80 \%$. Decrease in micelle availability and micellar structuring effectiveness, with interaction effects, explains the impact of microemulsion on foam stability.
\end{abstract}

Keywords Foam stability $\cdot$ Microemulsion $\cdot$ Enhanced oil recovery $\cdot$ Low tension gas · Oil-swollen micelles

\section{Introduction}

Surfactant-stabilized aqueous foams have recently received increased attention from the petroleum industry due to their excellent liquid mobility control potential for chemical enhanced oil recovery processes such as low tension gas (LTG) flooding. In the LTG process, a blend of surfactants is introduced to injected water to generate low interfacial tension microemulsions to mobilize oil and to stabilize and propagate foam to effectively displace mobilized oil (Jong et al. 2016).

During chemical injection, the different portions of the reservoir are subjected to a range of phase behavior as injection salinity (electrolyte concentration) is altered. As salinity is increased for a given system of surfactant, oil, and brine, the phase behavior will change from Winsor Type I to Type III to Type II (Winsor 1948), which correspond to an aqueous microemulsion phase with solubilized oil

Quoc Phuc Nguyen

quoc_p_nguyen@mail.utexas.edu

Stephen Yin-Chyuan Jong

sjong13@gmail.com

1 Center for Petroleum and Geosystems Engineering, University of Texas at Austin, 200 E Dean Keeton St. C0304, Austin, TX 78712, USA (within surfactant micelles) in equilibrium with excess oil, a bicontinuous middle phase microemulsion with solubilized oil and water in equilibrium with excess oil and excess water, and an oleic microemulsion phase with solubilized water (within surfactant micelles) in equilibrium with excess water, respectively. Recent work done by Jong et al. (2016) to study the effect of changing salinity environments on LTG performance indicates that varying microemulsion phase behavior has a strong impact on foam stability.

Foam (gas dispersed in water) stability is governed by film-scale phenomena such as dynamic disjoining pressure, capillary suction, and gravity drainage (Schramm and Wassmuth 1994). Liquid films separating a dispersed gas phase are subject to capillary pressure that promotes film thinning due to the higher pressure in the gas phase. For thin films, the capillary pressure is balanced by the disjoining pressure, which is the force per unit area exerted by a liquid film in excess of that in the bulk liquid and may result in either repulsion or attraction between the surfaces bounding the film, which in the case of foam are the gas bubbles between liquid lamellae (Schramm and Wassmuth 1994). The surface mobility of a foam film, which is significantly influenced by surface elasticity and surface viscosity, also contributes to the dynamics of film drainage and stability (Schramm and Wassmuth 1994). 
The addition of surfactant, which adsorbs at the gas-water interface, will stabilize foam by increasing the maximum of the disjoining pressure isotherm as well as giving rise to the Gibbs-Marangoni effect which resists interface deformation due to localized surface tension gradients. Addition of surfactant above critical micelle concentration (CMC) will not result in further aggregation of surfactant at the gas-water interface but instead contribute to micelle formation, which increases the pressure of the aqueous film beyond that in the bulk aqueous phase. This excess pressure is called structural disjoining pressure and is caused by micellar structuring when confined in an aqueous film. Micellar structuring has been demonstrated to result in delayed film thinning in a stepwise manner (Nikolov and Wasan 1989; Wasan et al. 1994; Shah 1981).

Theory and experimental work (Ross and Bramfitt 1957; Ross and Haak 1958; Lee et al. 2012, 2014a, b, 2016) indicates that oil-swollen micelles may affect micellar structuring and decrease foam stability compared to oil-free micelles due to reduced intramicellar repulsion. We postulate that the interaction of microemulsions and foams may be explained by the micelles, which are dispersed within an external phase to comprise microemulsions. We constrain the current work to Type I microemulsions, because their influence on foam stability may readily be compared to the oil-free foam stability case due to Type I microemulsions still being a predominantly aqueous phase with surfactant micelles (albeit oil-swollen) that provide structuring when confined to an aqueous film. Increasing salinity within the Type I region will produce a range of microemulsions which differ in readily quantifiable properties such as oil concentration and oil-swollen micelle diameter.

By observing the foam stability to varying microemulsions in combination bulk and confined (porous medium scale) foam tests and drawing upon understanding of microemulsion phase behavior, we aimed to develop a working theory based on film-scale interactions to explain the observed macroscopic phenomena. We hypothesized that microemulsion-foam interactions may be explained by the action of oil-swollen micelles within foam lamellae, which varies with salinity as micelle properties and concentration change.

\section{Experimental}

\section{Materials and phase behavior}

We screened several surfactant-crude oil systems for microemulsion and foam generation potential and a large Type I microemulsion window with significant oil solubilization. Our selected system consisted of a 1:1 ratio of $\mathrm{C}_{15-18}$ internal olefin sulfonate $\left(\mathrm{R}-\mathrm{CH}(\mathrm{OH})-\mathrm{CH}_{2}-\mathrm{CH}\left(\mathrm{SO}_{3}{ }^{-}\right)-\mathrm{R}^{\prime}(75 \%\right.$ molar concentration) and $\mathrm{R}-\mathrm{CH}=\mathrm{CH}-\mathrm{CH}\left(\mathrm{SO}_{3}{ }^{-}\right)-\mathrm{R}^{\prime}(25 \%$ molar concentration, where $\mathrm{R}+\mathrm{R}^{\prime}=\mathrm{C}_{12-15}$ ) and $\mathrm{C}_{16-17}$ alcohol 7-propoxy sulfate $\left(b \mathrm{C}_{16-17}-\left[\mathrm{CH}_{2}\left(\mathrm{CH}_{3}\right) \mathrm{CH}-\mathrm{O}\right]_{7}-\mathrm{SO}_{3}{ }^{-}\right)$ for our anionic surfactant blend and a light (37.1 API gravity), low viscosity $(3.1 \mathrm{cP})$ crude oil. The surfactants and crude oil were gifts from Stepan Company and Shell Global Solutions, respectively.

An aqueous stability test was conducted to identify the critical salinity ( $w t \% \mathrm{NaCl}$ ) above which surfactants precipitate. After the critical salinity was determined, $350.0 \mu \mathrm{L}$ of $4.0 \mathrm{wt} \%$ surfactant stock, $400.0 \mu \mathrm{L}$ of crude oil, and varying amounts of $14.0 \mathrm{wt} \% \mathrm{NaCl}$ and deionized water (resulting in $2.8 \mathrm{~mL}$ total aqueous solution with $0.5 \mathrm{wt} \%$ surfactant concentration) were dispensed into Fisherbrand $5.0 \mathrm{~mL}$ borosilicate serological pipettes to perform a salinity scan. The pipettes were heat-sealed and allowed to equilibrate in a convection oven at $35^{\circ} \mathrm{C}$ for 7 days and subjected to inversion six times per day to promote mixing. Phase behavior and aqueous stability results indicated Type I microemulsion phase behavior (Fig. 1) and aqueous stability up to $9.0 \mathrm{wt} \%$ $\mathrm{NaCl}$. Seven samples were chosen within the Type I range to represent varying degrees of oil solubilization. The maximum salinity chosen was $7.0 \mathrm{wt} \% \mathrm{NaCl}$ to avoid the transition zone between Type I and Type III microemulsions. Sample properties are listed in Table 1.

We followed the same procedure to produce more samples, substituting Fisherbrand borosilicate test tubes for pipettes and removing excess oil via suction before extracting the equilibrated microemulsion phase into secondary test tubes. Microemulsion extraction occurred from the bottom of the original test tubes and ceased when approximately $3.0 \mathrm{~mL}$ of sample remained to avoid taking any sample from near the original microemulsion-excess oil interface. Diluted samples for $2.0,4.5$, and $6.0 \mathrm{wt} \%$ salinity were also produced at 50.0, 25.0, and $12.5 \%$ original oil-swollen micelle concentration by mixing undiluted samples with equivalent salinity $\mathrm{NaCl}$ solutions to control micelle size while varying micelle concentration.

\section{Microemulsion characterization}

We characterized the oil-swollen micelles in each microemulsion sample according to particle size and concentration. Size measurements were made using a Malvern Zetasizer ZS, a dynamic light scattering (DLS) machine (Fig. 2). These measurements agree with theoretical calculations and previous measurements of droplet size for microemulsion systems (Huh 1979). Micelle size is in the range of 9.0-28.0 nm and increases with increasing salinity within the Type I region with a trend similar to oil solubilization ratio. This is expected, because the solubilization ratio and droplet size are linked by the propensity 
Fig. 1 Phase behavior scan from 0.0 to $9.0 \mathrm{wt} \% \mathrm{NaCl}$ in $1.0 \mathrm{wt} \%$ steps from left to right. Dark upper phase is oil and light lower phase is Type I microemulsion

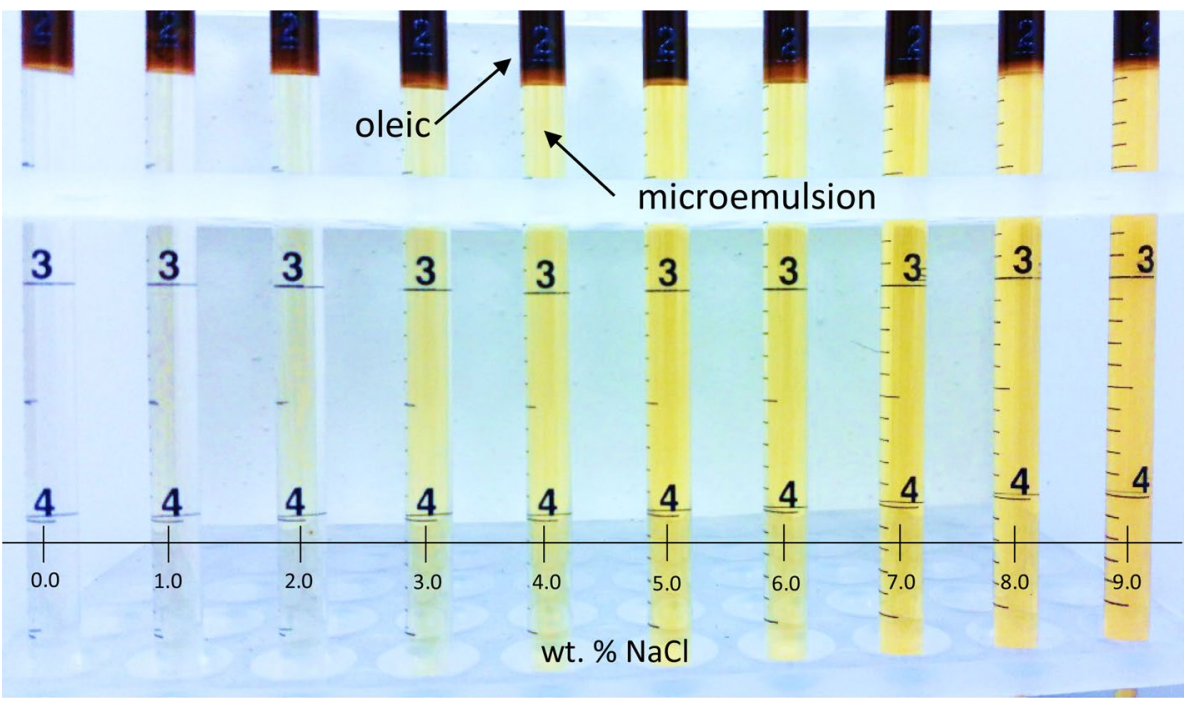

Table 1 Microemulsion sample properties

\begin{tabular}{lllll}
\hline Sample & $\begin{array}{l}\text { Salinity }(\mathrm{wt} \% \\
\mathrm{NaCl})\end{array}$ & $\begin{array}{l}\text { Oil concentration } \\
(\mathrm{v} / \mathrm{v} \%)\end{array}$ & $\begin{array}{l}\text { Oil-swollen micelle } \\
\text { diameter }(\mathrm{nm})\end{array}$ & $\begin{array}{l}\text { Viscosity at } 15 / \mathrm{s} \\
\text { and } 35^{\circ} \mathrm{C}(\mathrm{cP})\end{array}$ \\
\hline 1 & 2.0 & 0.93 & 9.30 & 0.86 \\
2 & 3.5 & 1.09 & 11.86 & 0.96 \\
3 & 4.5 & 1.57 & 13.80 & 0.95 \\
4 & 5.5 & 1.93 & 16.63 & 0.96 \\
5 & 6.0 & 2.41 & 18.26 & 0.98 \\
6 & 6.5 & 2.89 & 20.65 & 0.91 \\
7 & 7.0 & 3.92 & 27.08 & 0.91 \\
\hline
\end{tabular}

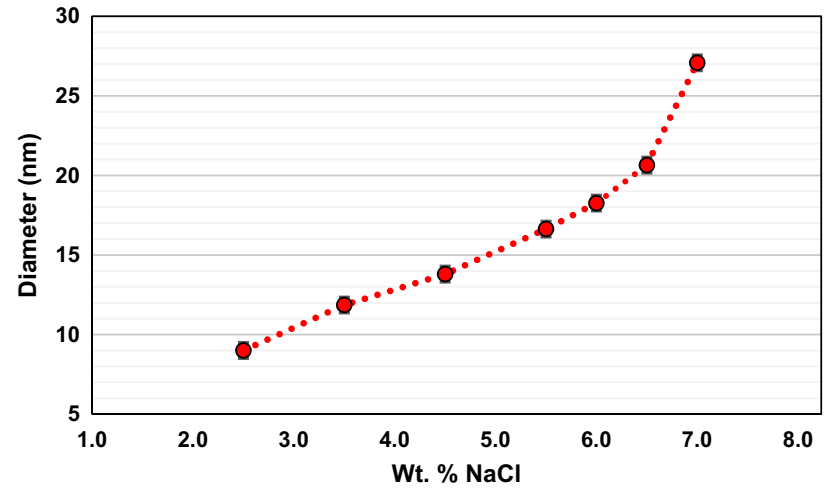

Fig. 2 Oil-swollen micelle size vs. salinity measured by dynamic light scattering

of surfactant to adsorb at oil-water interfaces, which is controlled by the ion concentration in the aqueous phase.

In a phase behavior scan, upward movement of the oil-water interface with increasing salinity corresponds to increasing oil solubilization. Measurement of the amount of oil remaining allows for the calculation of oil volume fraction in the aqueous phase. Table 1 includes the oil concentration in the aqueous phase for each sample.

Oil volume fraction in the aqueous phase is only an intermediate step, since it is determined by size and concentration of oil-swollen micelles. Therefore, we took the approach of normalizing the solubilized oil to the volume of oil-swollen micelles from DLS measurement, assuming approximate sphericity and relative monodispersity (DLS polydispersity for all samples was less than $20.0 \%$, indicating monodispersity), before normalizing to microemulsion volume (will increase with increase in oil solubilization). Finally, we divided the results for samples 1-7 (Table 1) by the result for sample 1, giving a relative indication of oilswollen micelle concentration for each sample.

The results in Fig. 3 demonstrate increasing micellar aggregation number with increasing neutral electrolyte addition, as decreased repulsion between hydrophilic head groups allows for closer packing due to compression of the electrical double layer, larger micelles, and greater oil solubilization per micelle (Rosen and Kunjappu 2012). This factor outweighs the simultaneous decrease in CMC with increasing neutral electrolyte addition to result in net decrease of oil-swollen micelles. 


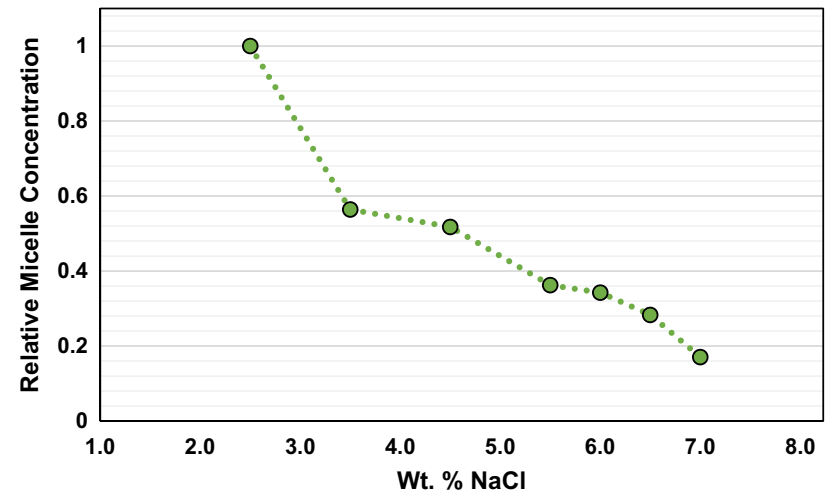

Fig. 3 Oil-swollen micelle concentration vs. salinity normalized to concentration for $2.5 \mathrm{wt} \% \mathrm{NaCl}$ (first sample)

\section{Surface tension and critical micelle concentration (CMC) measurements}

To determine whether microemulsion-foam interactions were due solely to the action of oil-swollen micelles within liquid lamellae or involved contribution from interactions with the air-water interface, we performed several series of surface tension measurements.

Our measurement system consisted of a ramé-hart F4 Series camera focused on a flat Teflon surface and placed opposite a 150w fiber optic illuminator. Measurements were made in the sessile drop mode via ramé-hart DropImage Advanced software, which captured the curvature of the drop and calculated surface tension via Young-Laplace methods.

We conducted a CMC measurement first with varying concentrations of surfactant in deionized water to gauge the maximum reduction in surface tension achievable with surfactant only. Afterwards, we measured surface tension of surfactant solutions well above CMC ( $0.5 \mathrm{wt} \%$ corresponding to the concentration in undiluted microemulsion samples) with varying salinities corresponding to the seven microemulsion samples (no oil was added) to gauge the base effect of increasing neutral electrolyte concentration. Finally, we measured the surface tension of the seven microemulsion samples to determine the presence or absence of free surfactant molecules in equilibrium with oil-swollen micelles.

\section{Dynamic foam stability setup}

We measured dynamic foam stability at the bulk scale to obtain a baseline for microemulsion-foam interactions and compare to microfluidic scale interactions. Our setup consisted of a $1000 \mathrm{~mL}$ Fisherbrand KiMax burette fixed vertically on a ring stand with $1 / 8^{\prime \prime}$ OD stainless steel tubing inserted in the center from the top onto a ring stand and placed the setup inside an oven at $35.0{ }^{\circ} \mathrm{C}$ (shown in Fig. 4). For each microemulsion sample, we dispensed $3.0 \mathrm{~mL}$ of

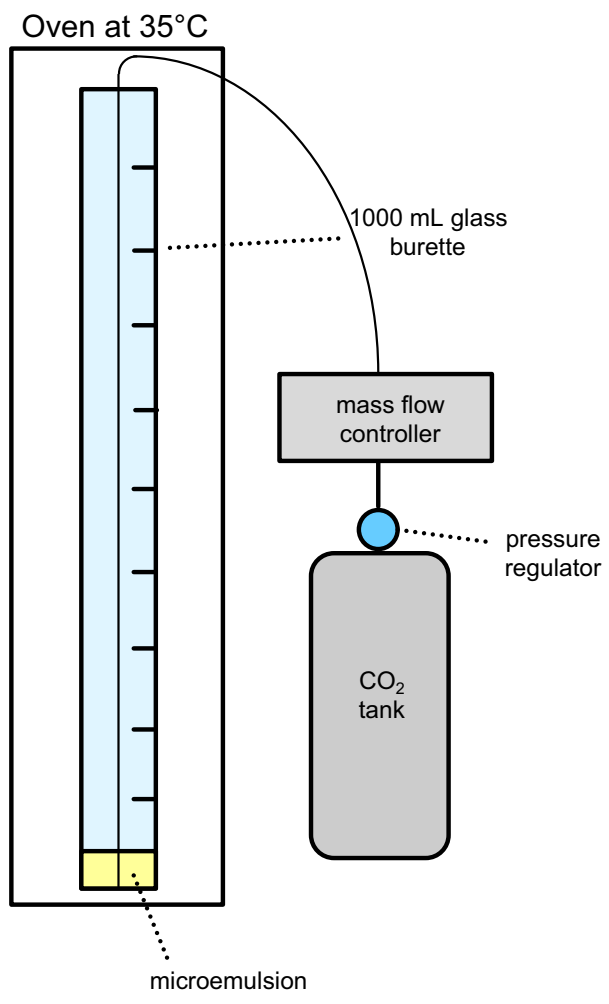

Fig. 4 Dynamic foam stability setup

the sample into the burette and bubbled $\mathrm{CO}_{2}$ into the sample from the base of the burette at a rate of 160.0 SCCM (standard cubic centimeters per minute). The experiment continued until the foam coalescence rate due to capillary suction and gravity drainage was equivalent to the foam generation rate due to bubbling $\mathrm{CO}_{2}$. We recorded this equilibrium height as a measure of the samples' dynamic foam stability before thoroughly rinsing the burette with deionized water and drying the burette with high pressure laboratory air.

This Bikerman-style experiment (Bikerman 1973) had the advantage of eliminating variance in the initial states between samples while also providing monodisperse generation of new foam bubbles. Since these experiments were done with pre-equilibrated Type I microemulsions free of excess or bulk oil, any change in foam stability was attributed to the presence of oil-swollen micelles.

\section{Microfluidic monitoring system setup}

To extend our findings from bulk foam studies to porous media applications, we developed an experimental setup to examine foam stability in a porous microflow model. A diagram of the setup is shown in Fig. 5. $\mathrm{CO}_{2}$ was routed from a tank maintained at $800 \mathrm{psi}$ and joined to a chemical solutionbearing Hamilton Gas Tight Syringe in a Chemyx Fusion Syringe Pump via a tee connection. $\mathrm{CO}_{2}$ was maintained at 
Fig. 5 Diagram of microfluidic setup with microscope images of the uniform and physical rock network chips

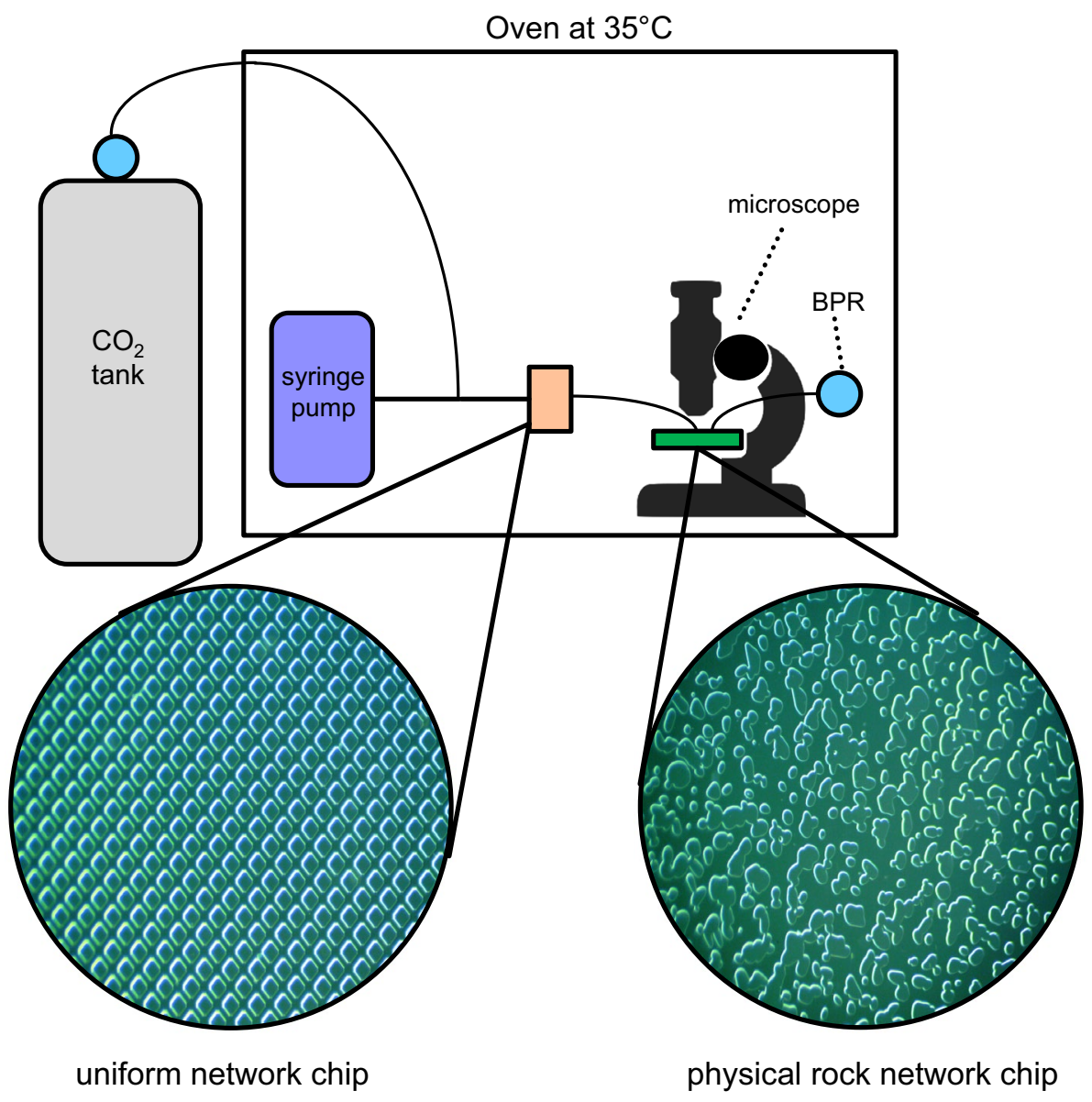

a constant upstream pressure of $25.0 \mathrm{psi}$ and chemical solution injection rate was set at $0.005 \mathrm{~mL} / \mathrm{min}$. Liquid and gas were co-injected into a Micronit borosilicate glass uniform microfluidic network $(45.0 \times 15.0 \mathrm{~mm}$ chip size, $50.0 \mu \mathrm{m}$ channel width, and $20.0 \mu \mathrm{m}$ channel depth) to promote mixing and monodisperse bubble generation before entering into a Micronit borosilicate glass microfluidic network based on a physical rock $(45.0 \times 15.0 \mathrm{~mm}$ chip size, $50.0 \mu \mathrm{m}$ channel width, and $20.0 \mu \mathrm{m}$ channel depth) (Fig. 5). We connected a 20.0 psi backpressure regulator to the outlet to avoid larger gas pressure fluctuations associated with a lower pressure range, thus keeping the gas injection at a constant 2.5 psi pressure drop across the chip. Pressure drop along the lines amounted to roughly 2.5 psi.

Each microemulsion sample was co-injected with $\mathrm{CO}_{2}$ to form foam in the physical rock network chip before shutting in the system. The relatively high rate of injection led to a uniform initial texture (number of foam bubbles per unit area) for all samples, since generation dominated coalescence. Decay was observed and recorded for $30 \mathrm{~min}$ via an AmScope MU300 Microscope Digital Camera connected to a laptop computer. After $30 \mathrm{~min}$, the chip was flushed extensively with deionized water to remove surfactant followed by dichloromethane to remove any oil left behind by microemulsions and 40 min of $\mathrm{CO}_{2}$ injection to ensure complete removal of dichloromethane before beginning the next experiment. We performed image analysis using ImageJ, and recorded the initial and final texture for each sample.

\section{Results and discussion}

\section{Surface tension measurements}

Once surfactant concentration in the aqueous phase has increased past CMC, minimal change is observed in surface tension, because surfactant has already reached maximum adsorption at the interface (Rosen and Kunjappu 2012). Additional surfactant leads to the formation of micelles that are in equilibrium with surfactant molecules (Mysels and Otter 1961a, b; Shah et al. 1996). These surfactant molecules ought to be present by definition at a concentration below $\mathrm{CMC}$; else, micelle formation is favored over independent molecules.

Type I microemulsions are comprised of oil-swollen surfactant micelles suspended in aqueous solution. These oil-swollen micelles only depress CMC slightly and are not found to adsorb at the air-water interface at equilibrium

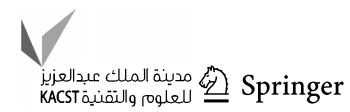


(Rosen and Kunjappu 2012). However, there is no previous work which indicates whether surfactant molecules exist in equilibrium with these oil-swollen micelles.

Therefore, we approached our surface tension measurements expecting one of two outcomes:

(a) Surface tension of microemulsions is higher than that of equivalent oil-free samples of varying salinity, indicating reduced availability or tendency of surfactant molecules to adsorb at the interface.

(b) Surface tension of microemulsions is equal to that of equivalent oil-free samples of varying salinity, indicating equivalent availability or tendency of surfactant molecules to adsorb at the interface.

Testing of the oil- and $\mathrm{NaCl}$-free surfactant solutions indicated a CMC of $0.046 \mathrm{wt} \%$ and minimum surface tension of 31.79 dynes $/ \mathrm{cm}$. Adding varying amounts of $\mathrm{NaCl}$ at $0.5 \mathrm{wt} \%$ surfactant concentration resulted in a small surface tension reduction to an average of 28.36 dynes/cm (Fig. 6), which was nearly constant across all samples ( 0.54 dynes/ $\mathrm{cm}$ standard deviation). Increased neutral electrolyte concentration in a surfactant solution leads to more efficient surfactant molecule adsorption at the air-liquid interface and lower surface tension (Ross and Bramfitt 1957), although, within this range, near-maximum adsorption at the air-liquid interface has already been achieved.

The same increase in neutral electrolyte concentration also leads to larger micelles, which can solubilize more oil. However, these were not found to have any additional effect on the air-liquid interface as the surface tension remained at an average of $\sim 27.93$ dynes $/ \mathrm{cm}(0.52 \mathrm{dynes} / \mathrm{cm}$ standard deviation) (Fig. 6). Based on these measurements and observations, it can be concluded that surfactant molecules are present and in equilibrium with oil-swollen micelles, which is in line with our second expected outcome. Therefore, we were confident in analyzing the proceeding experimental results in terms of intra-film micellar interactions rather than interfacial phenomena due to reduced surfactant molecule availability in the presence of oil-swollen micelles.

\section{Bulk foam stability testing}

The results of the bulk foam stability test (Fig. 7a) for the undiluted samples indicated a strong impact of microemulsion phase behavior on foam stability. We normalized the results to the control case of an oil-free sample whose stability was shown to be independent of salinity. The $2.0 \mathrm{wt} \% \mathrm{NaCl}$ case demonstrated a decrease of around $35 \%$ compared to the control case. As the salinity increased from $2.0 \mathrm{wt} \% \mathrm{NaCl}$ to $7.0 \mathrm{wt} \% \mathrm{NaCl}$ within the Type I microemulsion region, the dynamic foam stability decreased more than $90.0 \%$, as measured by equilibrium foam column height. The magnitude of foam stability reduction is monotonically decreasing with increasing salinity with the most dramatic change of $\sim 70.0 \%$ coming between 2.0 and $3.5 \mathrm{wt} \% \mathrm{NaCl}$. The salinity transition from 2.0 to $7.0 \mathrm{wt} \% \mathrm{NaCl}$ corresponded to increasing overall oil solubilization ( $0.93-3.92 \%$ concentration) and increasing micelle size $(9.0-28.0 \mathrm{~nm}$ in diameter) due to decreasing repulsion between hydrophilic head groups of surfactant molecules (Rosen and Kunjappu 2012), which led to decreasing micelle concentration (Fig. 3). Lee et al. (2014b, 2016) found that sodium dodecyl sulfate (SDS) solutions with solubilized $n$-dodecane resulted in less stable foam compared to oil-free solutions, because oil
Fig. 6 Surface tension measurement for oil-free salinity scan and microemulsion samples

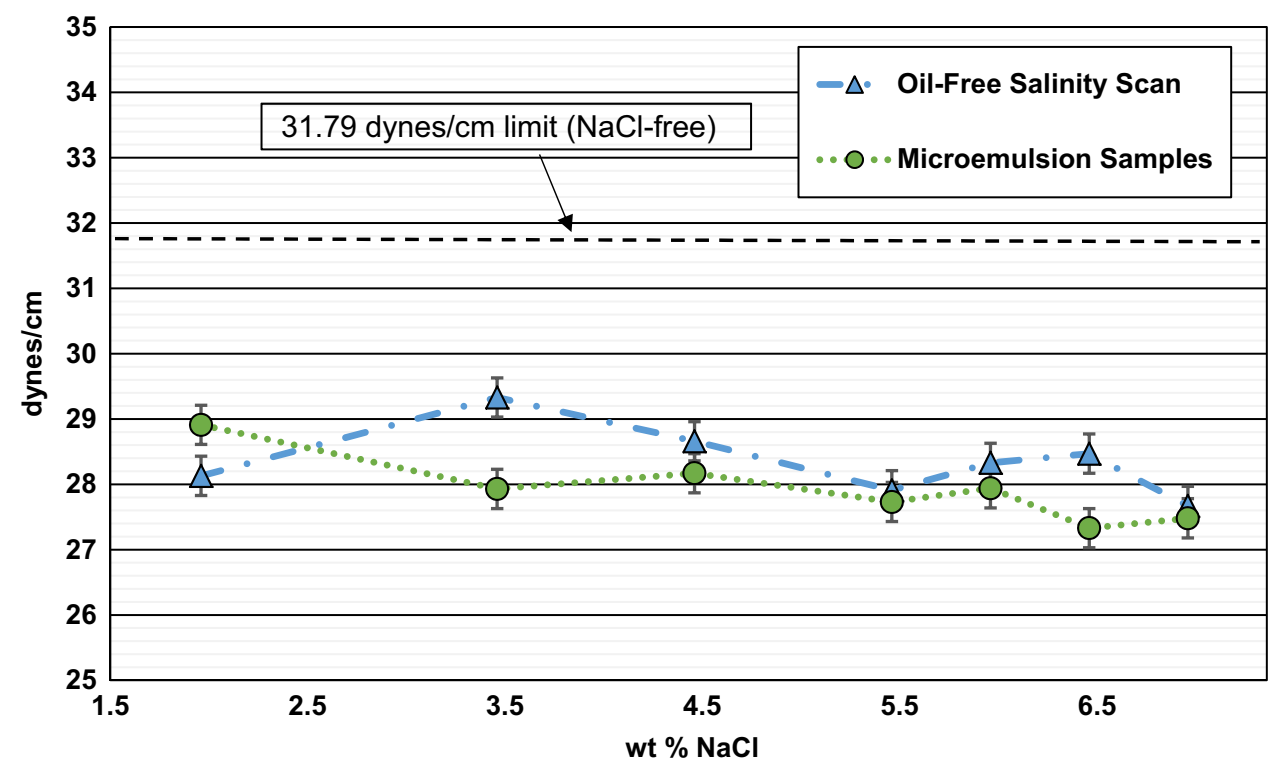




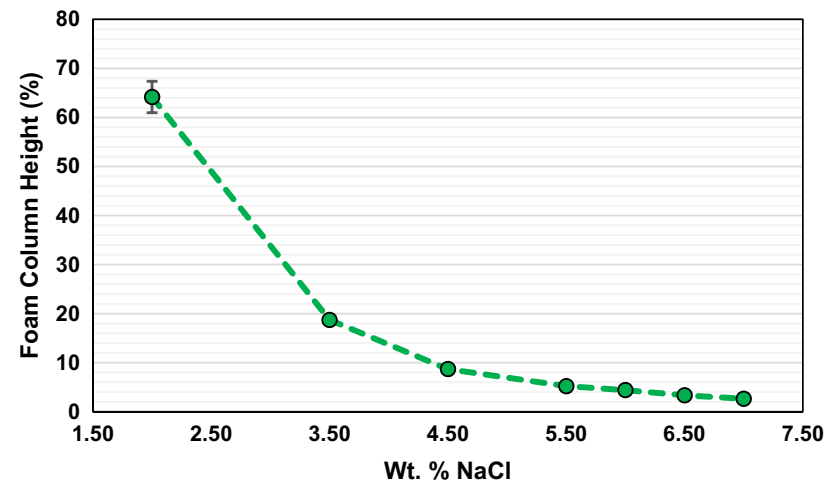

(a)

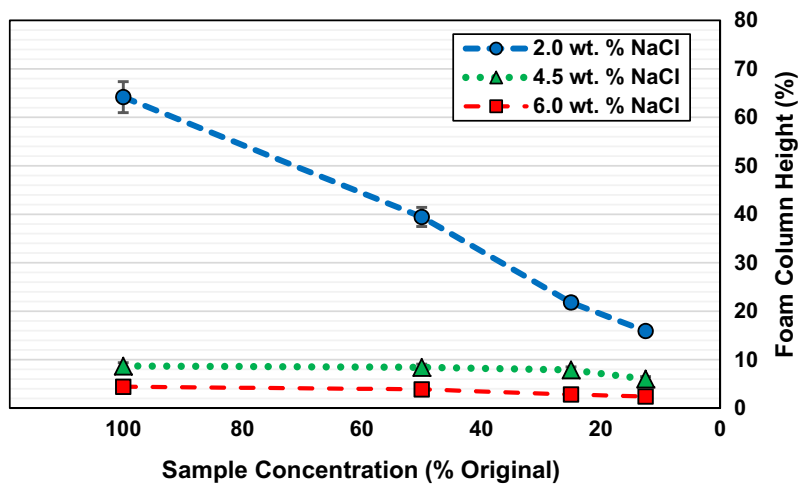

(b)

Fig. 7 Results of bulk foam stability testing, equilibrium foam column height vs. sample salinity normalized to equilibrium foam column height of oil-free sample for $\mathbf{a}$ undiluted and $\mathbf{b}$ diluted samples

solubilization reduced intramicellar repulsion. We ruled out the significance of the effect of bulk microemulsion viscosity on film drainage due to the fact that the stability decreases despite microemulsion viscosity remaining relatively constant with varying salinity (Table 1).

Therefore, the factors concerning oil-swollen micelles which directly impact foam stability are:

1. decreasing micelle concentration with increasing salinity;

2. increasing micelle size with increasing salinity;

3. decreasing intramicellar repulsion with increasing oil solubilization.

The $70.0 \%$ decrease in foam stability from $2.0 \mathrm{wt} \%$ $\mathrm{NaCl}$ to $3.5 \mathrm{wt} \% \mathrm{NaCl}$ (Fig. 7a) corresponded to the $50.0 \%$ decrease in micelle concentration (Fig. 3) compounded with decreasing intramicellar repulsion and increasing micelle size. However, the nearly $50.0 \%$ decrease in foam stability from 3.5 to $4.5 \mathrm{wt} \% \mathrm{NaCl}$ corresponded to only an $8.2 \%$ decrease in micelle concentration, so, here, the effects of decreased intramicellar repulsion and increased micelle size are more important. This indicated a non-linear relationship between foam stability and each of these three factors, and possible interaction between the factors themselves.

Our dilution tests controlled oil-swollen micelle size while varying concentration to clarify the individual contributions of these factors. Surface tension measurements showed no significant difference between diluted and undiluted samples, which allowed for continued analysis based on the action of oil-swollen micelles within the film rather than interfacial effects. The results shown in Fig. 7b demonstrated that, for each fixed size, diluting the sample reduced the foam stability. It should be noted that these dilutions $(50.0 \%)$ at most resulted in a commensurate (50.0\%) decrease in foam stability, and for most cases resulted in less than $50.0 \%$ decrease in foam stability. This is because micellar structuring affects foam stability in a non-linear fashion (Nikolov and Wasan 1989). The small changes in foam stability under dilution for 4.5 and $6.0 \mathrm{wt} \% \mathrm{NaCl}$ were due to the initially low micelle concentration in those samples, which would lead to less complex stratification and less penalty for dilution. Therefore, oil-swollen micelles exhibit less effective micellar structuring compared to oil-free micelles, but still contribute to a more stable foam by their presence rather than their absence.

\section{Microfluidic decay testing}

The transition to the porous microflow model allowed for the examination of foam stability in a much more confined medium. In particular, the foam in the microflow model differed from bulk foam, because it had two-dimensional connectivity of Laplace borders, was shaped by porous media, and was subject to higher capillary pressures due to the minute flow channels in a porous medium. In addition, the mode of testing was static decay rather than the dynamic mode of our Bikerman-style tests. The static decay test reproduced the phenomenon of foam decay in an enhanced oil recovery scenario far away from the injection well(s). In the far-field, mobile foam propagates slowly and a significant portion remains trapped. Comparing the trends of foam stability testing at different scales and by different methods allowed for the confirmation of the impact of oil-swollen micelles on foam stability in a porous medium.

Both the undiluted (Fig. 8a) and diluted (Fig. 8b) sample tests held to the general trend observed in the bulk foam stability testing, with foam stability decreasing as sample salinity was increased from 2.0 to $7.0 \mathrm{wt} \%$ and also as sample concentration was decreased from 100.0 to $12.5 \%$.

The similarity in the trends for both the undiluted and diluted samples at bulk and microfluidic scales improved our confidence in the previous interpretation concerning microemulsion-foam interactions. We noted that the relative importance of oil-swollen micelle concentration, micelle size, and intramicellar repulsion varied depending on the current state

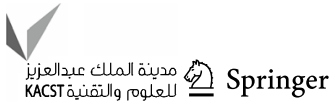




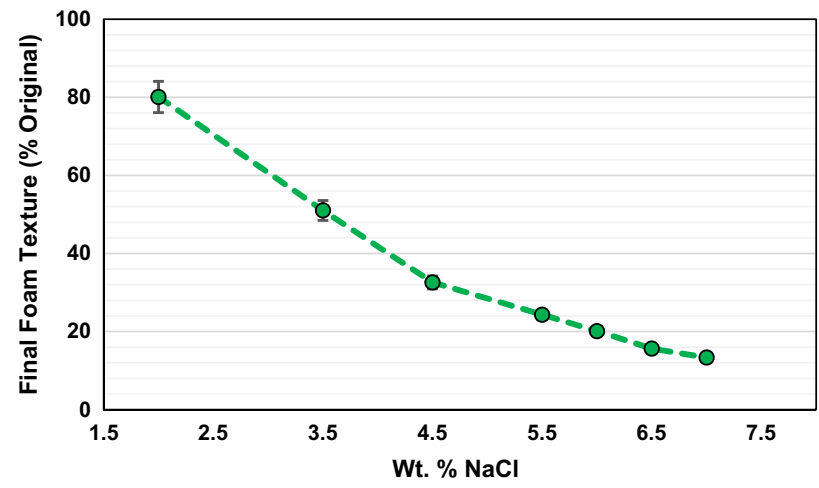

(a)

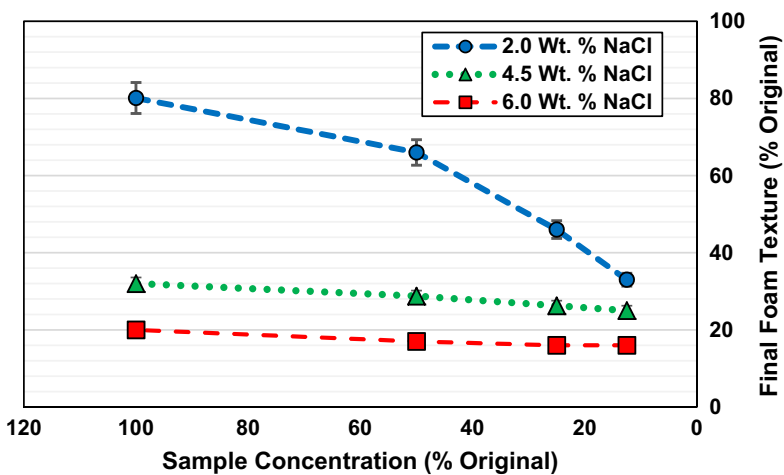

(b)

Fig. 8 Percentage of original texture in the microfluidic chip vs. sample salinity under static decay testing for $\mathbf{a}$ undiluted and $\mathbf{b}$ diluted samples

of each individual factor, confirming the presence of interaction effects at the porous medium scale in accordance with observations made at the bulk scale.

Reduced intramicellar repulsion with increasing salinity of Type I microemulsions leads to less resistance to film thinning, but it is not immediately clear how larger, sparser micelles contribute to foam stability. Lee et al. (2014a) have shown that as film thickness decreases, micellar structuring results in an ordered aggregation which resists film thinning, and based on our work, this viscosifying structure becomes less effective with larger, sparser oil-swollen micelles. Figure 9 presents a conceptual schematic of the impact of oilswollen micelles on foam stability.

\section{Conclusions}

This study demonstrates the significant effect of microemulsion on foam stability with the following key findings:

- Bulk foam dynamic stability and microfluidic foam static stability decreased approximately 95 and $85 \%$, respectively, in the presence of Type I microemulsion.

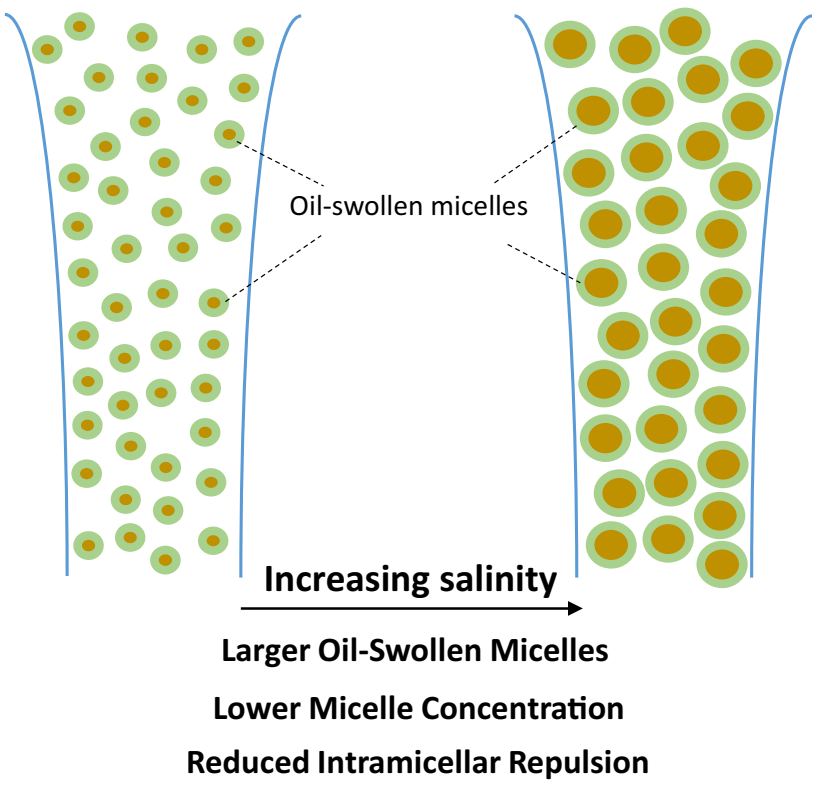

Fig. 9 Transition in micelles and micellar structuring with increasing salinity of Type I microemulsions

Foam stability decreased most significantly for the initial increase in salinity from 2.0 to $3.0 \mathrm{wt} \% \mathrm{NaCl}$ and then decreased more gradually as salinity was increased.

- This decrease in foam stability was due to the action of oil-swollen micelles within liquid lamellae, which exhibit impaired micellar structuring as neutral electrolyte concentration increases due to reduced availability, increased size, and decreased intramicellar repulsion. Interaction between effects may be significant, i.e., the relationship between foam stability and a certain factor is dependent on the present state of the other two factors, as well.

- Dilution testing showed that although oil-swollen micelles were not as effective as oil-free micelles in stabilizing foam, their presence still contributed to micellar structuring that resisted film drainage.

- Free surfactant molecules exist and are available to aggregate at the air-water interface in equilibrium with oil-swollen micelles in Winsor Type I microemulsions and this held true not only for the original $0.5 \mathrm{wt} \%$ surfactant solutions, but even for solutions with concentrations as low as $0.0625 \mathrm{wt} \%$ under dilution.

In the LTG process, effective oil mobilization due to ultralow oil-water IFT conditions occurs in the middle of the Type III microemulsion salinity range, but this study shows that effective oil displacement due to strong foam mobility control would occur in the lower salinity range of Type I microemulsion. Therefore, an LTG process design must account for the dramatic change in microemulsion 
environment required to facilitate strong foam mobility control and effective displacement of mobilized oil.

This work serves as a foundation for modeling the effect of microemulsion on foam stability according to three key factors which change with salinity. Future efforts will focus on developing a generalized model for microemulsion-foam interactions and extending the theories proposed here to non-equilibrium, transient oil displacement processes at both the microfluidic and core scale with the ultimate goal of incorporating this knowledge for field-scale LTG performance prediction.

\section{References}

Bikerman JJ (1973) Foams. Springer, Berlin

Huh C (1979) Interfacial tensions and solubilizing ability of a microemulsion phase that coexists with oil and brine. J Colloid Interface Sci 71(2):408-426

Jong S, Nguyen NM, Eberle CM, Nghiem LX, Nguyen QP (2016) Low tension gas flooding as a novel EOR method: an experimental and theoretical investigation. In: SPE 179559 presented at SPE improved oil recovery conference, Tulsa, Oklahoma, USA, 11-13 April 2016

Lee J, Nikolov AD, Wasan DT (2012) Stability of aqueous foams in the presence of oil: on the importance of dispersed vs solubilized oil. Ind Eng Chem Res 52(1):66-72

Lee J, Nikolov AD, Wasan DT (2014a) Effects of micellar structuring and solubilized oil on the kinetic stability of aqueous foams. Ind Eng Chem Res 53(49):18891-18899

Lee J, Nikolov AD, Wasan DT (2014b) Surfactant micelles containing solubilized oil decrease foam film thickness stability. J Colloid Interface Sci 415:18-25

Lee J, Nikolov AD, Wasan DT (2016) Stepwise thinning dynamics of a foam film formed from an anionic micellar solution. J Colloid Interface Sci 487:214-222

Mysels KJ, Otter RJ (1961a) Thermodynamic aspects of mixed micelles-application to an empirically established equilibrium. J Colloid Sci 16(5):474-480
Mysels KJ, Otter RJ (1961b) Conductivity of mixed sodium decyl and dodecyl sulfates-the composition of mixed micelles. J Colloid Sci 16(5):462-473

Nikolov AD, Wasan DT (1989) Ordered micelle structuring in thin films formed from anionic surfactant solutions: I. experimental. J Colloid Interface Sci 133(1):1-12

Rosen MJ, Kunjappu JT (2012) Surfactants and interfacial phenomena, 4 th edn. Wiley, New York

Ross SJ, Bramfitt TH (1957) Inhibition of foaming. VIII. Changes in electrical conductivity of colloidal electrolyte solutions on addition of non-ionic foam stabilizers and foam inhibitors. J Phys Chem 61(10):1261-1265

Ross SJ, Haak RM (1958) Inhibition of foaming. IX. Changes in the rate of attaining surface tension equilibrium in solutions of surface-active agents on addition of foam inhibitors and foam stabilizers. J Phys Chem 62(10):1260-1264

Schramm LL, Wassmuth F (1994) Foams: basic principles. In: Foams: fundamentals and applications in the petroleum industry, ACS advances in chemistry series, vol 242. American Chemical Society, Washington, DC, pp 3-45

Shah DO (1981) Fundamental aspects of surfactant-polymer flooding process. In: Presented at European symposium on enhanced oil recovery, Bournemouth, UK, 21-23 September 1981

Shah DO, Oh SG, Huibers PDT (1996) Pressure-jump studies on micellar relaxation time and its effect on various technological processes. In: Chattopadhyay AK, Mittal KL (eds) Surfactants in solution. Marcel Dekker, Inc., New York

Wasan DT, Koczo K, Nikolov AD (1994) Mechanisms of aqueous foam stability and antifoaming action with and without oil. In: Schramm LL (ed) Foams: fundamentals and applications in the petroleum industry, ACS advances in chemistry series, vol 242. American Chemical Society, Washington, DC, pp 47-114

Winsor PA (1948) Hydrotropy, solubilization and related emulsification processes. Trans Faraday Soc 44:376-398

Publisher's Note Springer Nature remains neutral with regard to jurisdictional claims in published maps and institutional affiliations. 